\title{
Aneurisma verdadeiro isolado de artéria femoral profunda associado a doença arterial periférica: relato de caso
}

\author{
True isolated deep femoral artery aneurysm associated with peripheral artery disease: \\ case report
}

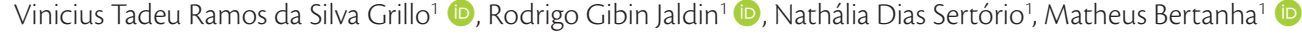 \\ Marcone Lima Sobreira' (D), Ricardo de Alvarenga Yoshida ${ }^{1}$ (D), Winston Bonetti Yoshida ${ }^{1}$
}

\begin{abstract}
Resumo
Os aneurismas verdadeiros de artéria femoral profunda são extremamente raros, representando cerca de 0,5\% do total dos aneurismas periféricos. Neste relato, descrevemos um paciente de 79 anos de idade, sexo masculino, com histórico de abordagem cirúrgica prévia convencional devido a aneurisma de aorta abdominal, que deu entrada no Serviço de Cirurgia Vascular do Hospital das Clínicas com quadro de claudicação intermitente de membros inferiores. Foi realizado eco-Doppler colorido arterial do membro inferior direito, que revelou doença arterial periférica femoro-poplítea e infrapatelar. A angiotomografia computadorizada identificou oclusão aortoilíaca e do enxerto bifurcado desde o segmento infrarrenal da aorta, além de aneurisma de artéria femoral profunda de 3,7 x 3,5 cm de diâmetro com $7 \mathrm{~cm}$ de extensão. Procedeu-se com a ressecção do aneurisma e revascularização da artéria femoral profunda por interposição de prótese de Dacron ${ }^{\circledR}$ e reimplante de artéria femoral superficial na prótese. Portanto, nos casos de aneurisma de artéria femoral profunda concomitante a doença arterial periférica, deve-se atentar para revascularização e perfusão adequada do membro inferior.
\end{abstract}

Palavras-chave: artéria femoral; aneurisma; doença arterial periférica; procedimentos cirúrgicos vasculares.

\begin{abstract}
True deep femoral artery aneurysms are extremely rare, accounting for about $0.5 \%$ of all peripheral aneurysms. In this report, we describe a 79-year-old male patient with a history of prior abdominal aortic aneurysm surgery via a conventional approach who was admitted to the vascular surgery service at the Hospital das Clínicas with intermittent claudication of the lower limbs. Arterial color-Doppler ultrasonography of the right lower limb was performed, revealing peripheral arterial disease of the femoral--popliteal and infrapatellar segments. Computed tomography angiography identified aortoiliac and bifurcated graft occlusion from the infrarenal segment of the aorta, in addition to a deep femoral artery aneurysm with diameters of $3.7 \mathrm{~cm} \times 3.5 \mathrm{~cm}$ and length of $7 \mathrm{~cm}$. Resection of the aneurysm was followed by revascularization of the deep femoral artery by interposition of a Dacron ${ }^{\circledR}$ graft and reimplantation of the superficial femoral artery into the graft. In cases of deep femoral artery aneurysms with concomitant peripheral arterial disease, it is important to ensure revascularization and adequate perfusion of the lower limb.
\end{abstract}

Keywords: femoral artery; aneurysm; peripheral arterial disease; vascular surgical procedures.

Como citar: Grillo VTRS, Jaldin RG, Sertório ND, et al. Aneurisma verdadeiro isolado de artéria femoral profunda associado a doença arterial periférica: relato de caso. J Vasc Bras. 2021;20:e20200220. https://doi.org/10.1590/1677-5449.200220

\footnotetext{
1 Universidade Estadual Paulista "Júlio de Mesquita Filho" - UNESP, Hospital das Clínicas da Faculdade de Medicina de Botucatu - HC-FMB, Serviço de Cirurgia Vascular e Endovascular, Botucatu, SP, Brasil. Fonte de financiamento: Nenhuma.

Conflito de interesse: Os autores declararam não haver conflitos de interesse que precisam ser informados.

Submetido em: Novembro 24, 2020. Aceito em: Janeiro 07, 2021
}

O estudo foi realizado no Hospital das Clínicas, Faculdade de Medicina de Botucatu, Universidade Estadual "Júlio de Mesquita Filho" (UNESP), Botucatu, SP, Brasil.

Copyright $₫ 2021$ Os autores. Este é um artigo publicado em acesso aberto (Open Access) sob a licença Creative Commons Attribution, que permite uso, distribuição e reprodução em qualquer meio, sem restrições desde que o trabalho original seja corretamente citado. 


\section{INTRODUÇÃO}

Considera-se aneurisma isolado da artéria femoral profunda (AFP) aquele que não possui continuidade com o aneurisma da artéria femoral comum (AFC). Os aneurismas verdadeiros de AFP são extremamente raros, representando cerca de $0,5 \%$ do total dos aneurismas periféricos e 1-2,6\% dos aneurismas femorais $^{1-5}$. A etiologia é, em sua maioria, aterosclerótica e são mais comuns em homens (92\%), com idade média de aproximadamente $70 \operatorname{anos}^{6-8}$.

Diante de sua raridade e da escassez de estudos da evolução natural dos aneurismas de AFP, não há, na literatura atual, critérios precisos para sua indicação cirúrgica nem qual seria a melhor técnica de tratamento ${ }^{4,5,7,9}$. Neste estudo, relatamos uma opção de tratamento cirúrgico em paciente portador de aneurisma isolado de AFP associado à doença arterial periférica (DAP). O protocolo foi aprovado pelo Comitê de Ética de nossa instituição (parecer número 4.699.360).

\section{RELATO DE CASO}

Paciente de 79 anos de idade, sexo masculino, branco, ascendência japonesa, avaliado no Serviço de Cirurgia Vascular do Hospital das Clínicas com queixa de dor em quadril direito e claudicação intermitente de membros inferiores para 100 metros, sem dor em repouso ou lesão trófica, que evoluiu com piora da intensidade há aproximadamente 4 meses. Histórico de hipertensão arterial sistêmica, ex-tabagista (cinco maços-ano), tendo cessado há mais de 50 anos, foi submetido à cirurgia de interposição arterial aortoilíaca com prótese Dacron ${ }^{\circledR}$ bifurcada por aneurisma de aorta infrarrenal em outro serviço há 16 anos, que se apresentava com oclusão há cinco anos.

Ao exame físico, apresentava-se em bom estado geral, com cicatriz abdominal de laparotomia prévia. Na região inguinal direita, palpava-se abaulamento de consistência fibroelástica e pulsátil. Os pulsos femorais apresentavam-se diminuídos bilateralmente $(2+/ 4+)$, e os pulsos poplíteos e distais estavam ausentes. Foram mensurados os índices tornozelo-braquial (ITB) bilateralmente: à direita, a artéria tibial anterior não tinha fluxo identificável ao Doppler ultrassom, e as artérias tibial posterior e fibular estavam com o ITB de 0,42 e 0,21 , respectivamente. À esquerda, registrou-se ITB de artérias tibial anterior, tibial posterior e fibular de $0,47,0,63$ e 0,42 , respectivamente. Não havia presença de lesões tróficas.

Realizou-se ainda estudo complementar por ecoDoppler colorido (EDC) arterial de membro inferior direito, que identificou estenoses e suboclusões segmentares das artérias infrapatelares. $\mathrm{O}$ paciente foi submetido à angiotomografia computadorizada
(ATC) de abdômen e pelve, sendo identificada oclusão aortoilíaca (intraprótese de Dacron ${ }^{\circledR}$ ) desde o segmento infrarrenal da aorta. As AFCs apresentavam-se pérvias por reenchimento por circulação colateral de calibre considerável, oriunda das artérias epigástricas inferiores e torácicas internas. Identificou-se aneurisma de AFP de 3,7 x 3,5 cm de diâmetro (anteroposterior e laterolateral, respectivamente) $\mathrm{com} 7 \mathrm{~cm}$ de extensão (Figura 1) e com trombos em seu interior. Foi reconstruída a imagem para avaliação das relações anatômicas do aneurisma (Figura 2).

Indicada a abordagem cirúrgica, realizou-se avaliação de risco pré-operatório, que classificou o paciente como risco moderado risco para complicações cardiovasculares ${ }^{10}$. Antevendo a dificuldade relacionada

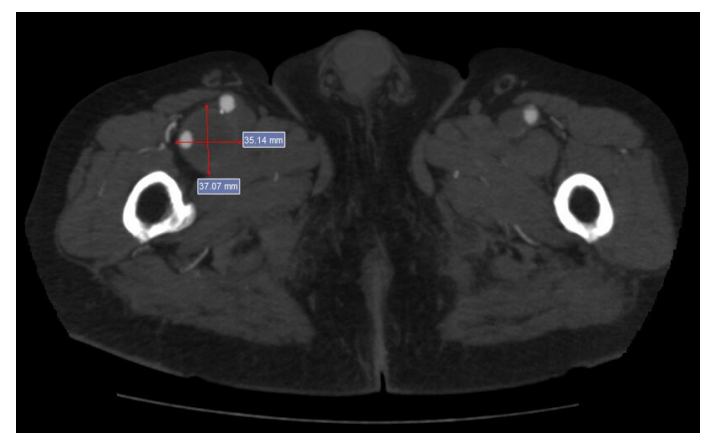

Figura 1. Angiotomografia computadorizada (ATC) em corte axial da coxa proximal, com identificação da dilatação aneurismática da artéria femoral profunda (AFP) com dimensões de 3,7 cm (anteroposterior) e 3,5 cm (laterolateral), com trombos em seu interior.



Figura 2. Reconstrução tridimensional de angiotomografia computadorizada (ATC) com referências ósseas, observando-se reenchimento de artéria femoral comum (AFC) por colaterais e dilatação aneurismática da artéria femoral profunda (AFP), sem envolvimento da AFC e artéria femoral superficial (AFS). 
à técnica e à tática, foi descartada a abordagem endovascular devido ao histórico do pós-operatório de revascularização aortoilíaca com oclusão da prótese associado a DAP dos membros inferiores.

$\mathrm{O}$ acesso cirúrgico deu-se por inguinotomia longitudinal ampla, discretamente lateralizado em relação à abordagem convencional, com identificação e isolamento da AFC e artéria femoral superficial (AFS), que se encontravam ectasiadas, e da AFP, a qual apresentava aneurisma que iniciava após a sua origem na bifurcação, seguia trajeto póstero-inferior por aproximadamente $7 \mathrm{~cm}$ e normalização do calibre após (Figura 3). Foram realizadas ressecção do aneurisma e revascularização da AFP. Não foram consideradas técnicas como embolização ou ligadura, dada a coexistência de ateromatose difusa da AFS e oclusão de artérias da perna, considerando a AFP como importante fonte de circulação colateral para perfusão do membro. Após isolamento seguro dos segmentos arteriais proximal e distal e posicionamento de clampes atraumáticos tipo DeBakey, procedeu-se a ressecção do trajeto aneurismático. Optou-se, inicialmente, por revascularização da AFP para diminuir o tempo de isquemia do membro, visto que, pela associação com DAP, a AFP era a responsável pela perfusão distal. Na sequência, procedeu-se com a interposição término-terminal de prótese de Dacron ${ }^{\circledR} 8 \mathrm{~mm}$ entre AFC e colo distal saudável de AFP e posterior secção e reimplante da AFS na prótese, garantindo trajeto de melhor caimento anatômico (Figura 4). Terminado o procedimento, foram palpados pulsos pré e pósanastomoses, sem a presença de assimetria ou frêmito. Como o paciente não era claudicante nem estava isquêmico, optou-se por não realizar a revascularização distal, convencional ou endovascular, pois poderia aumentar a morbimortalidade do procedimento. Frente à grande área de espaço-morto provocado pelas dimensões do aneurisma, optou-se por alocação de dreno a vácuo no tecido celular subcutâneo. A peça cirúrgica (Figura 5) foi enviada para análise anatomopatológica, que revelou provável origem aterosclerótica, com placa calcificada e trombo fibrinoleucocitário no lúmen (Figuras 6 e 7). O paciente apresentou boa evolução, o dreno foi retirado no segundo dia de pós-operatório (DPO), recebendo alta hospitalar no $5^{\circ} \mathrm{DPO}$. Nos retornos ambulatoriais de 14 e 30 dias, o paciente apresentava cicatrização total da ferida operatória, sem queixas álgicas, vasculares ou neurológicas. No exame físico, mantinha os pulsos femorais palpáveis bilateralmente $(2+/ 4+)$, e os pulsos poplíteos e distais estavam ausentes. Apresentava-se com extremidade do membro inferior direito quente e tempo de enchimento capilar menor do que 3 segundos.

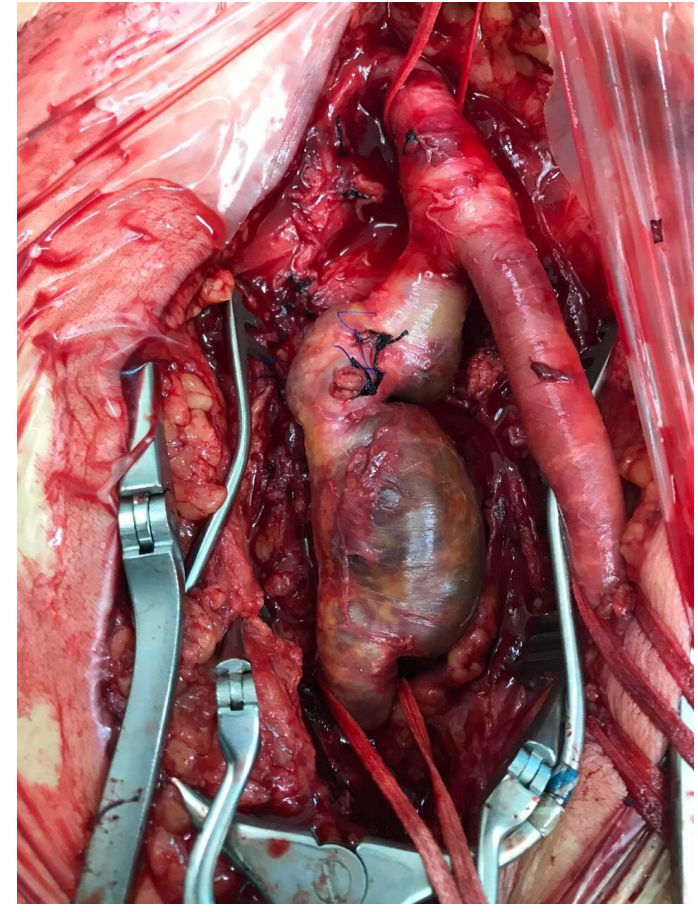

Figura 3. Resultado da dissecção cirúrgica, após reparo proximal da artéria femoral comum (AFC) e distal da artéria femoral superficial (AFS) e artéria femoral profunda (AFP) após a dilatação. Nota-se ligadura de ramos originados do aneurisma da AFP.

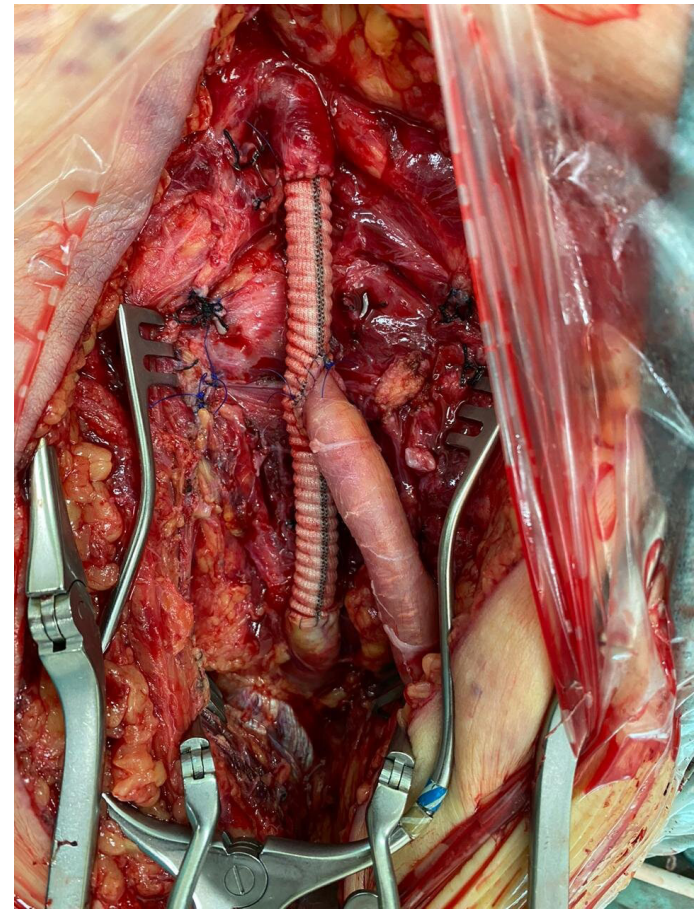

Figura 4. Resultado da revascularização, com interposição da prótese de Dacron ${ }^{\circledR}$ e anastomose proximal com artéria femoral comum (AFC) e distal com artéria femoral profunda (AFP), associado a reimplante da artéria femoral superficial (AFS) na prótese. 


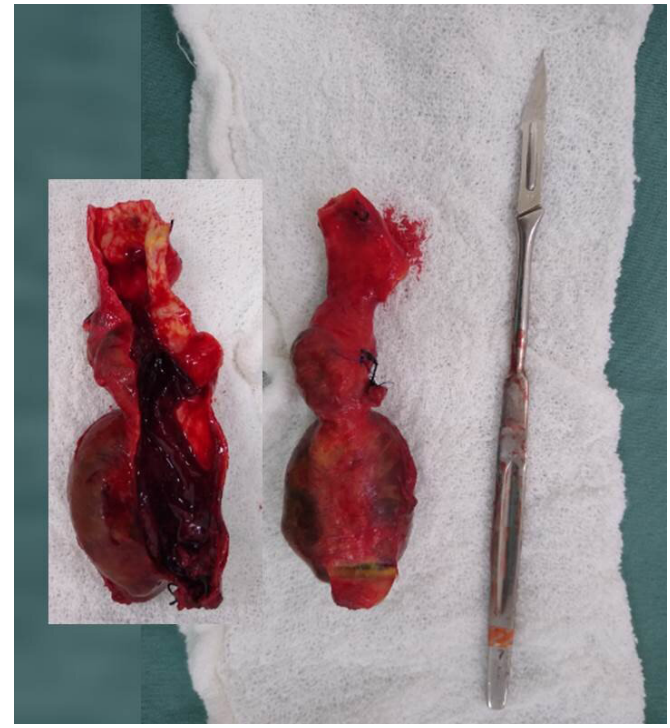

Figura 5. Peça cirúrgica. Morfologia do aneurisma e sua relação com a dimensão do cabo de bisturi número 7 e lâmina 11 . No destaque: morfologia do aneurisma após abertura, observandose trombos intraluminais.

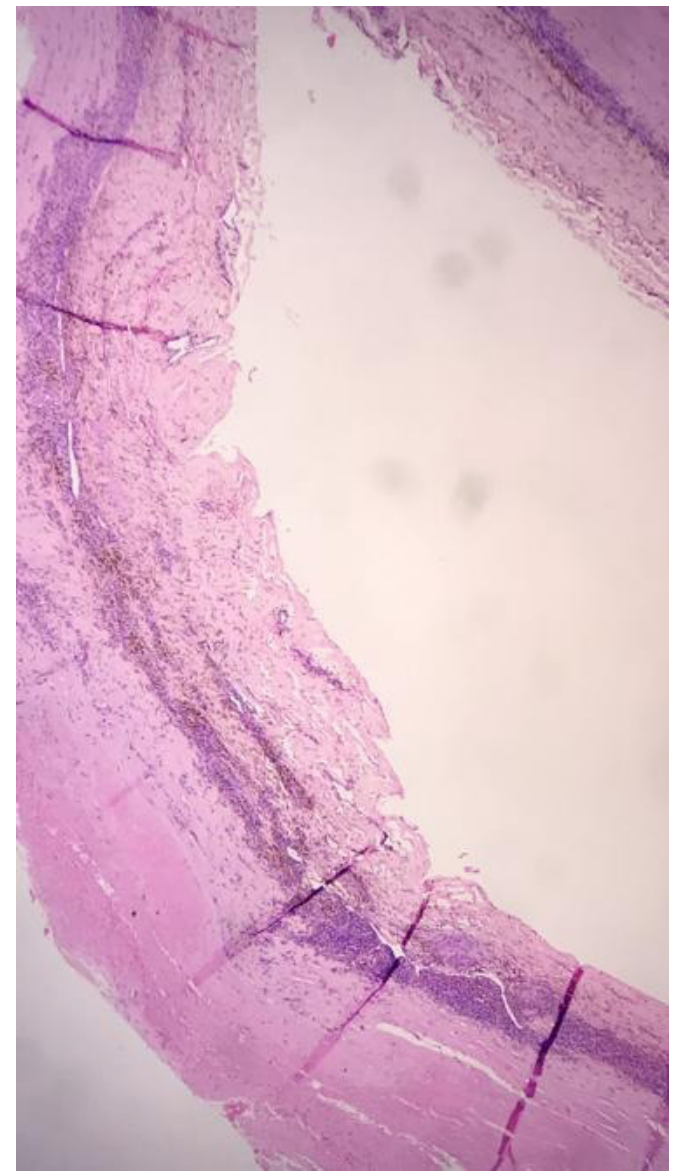

Figura 6. Análise histopatológica. Coloração H\&E: escala de $\times 400$. Artéria com lúmen dilatado, camada média degenerada e adventícia contendo inflamação crônica.

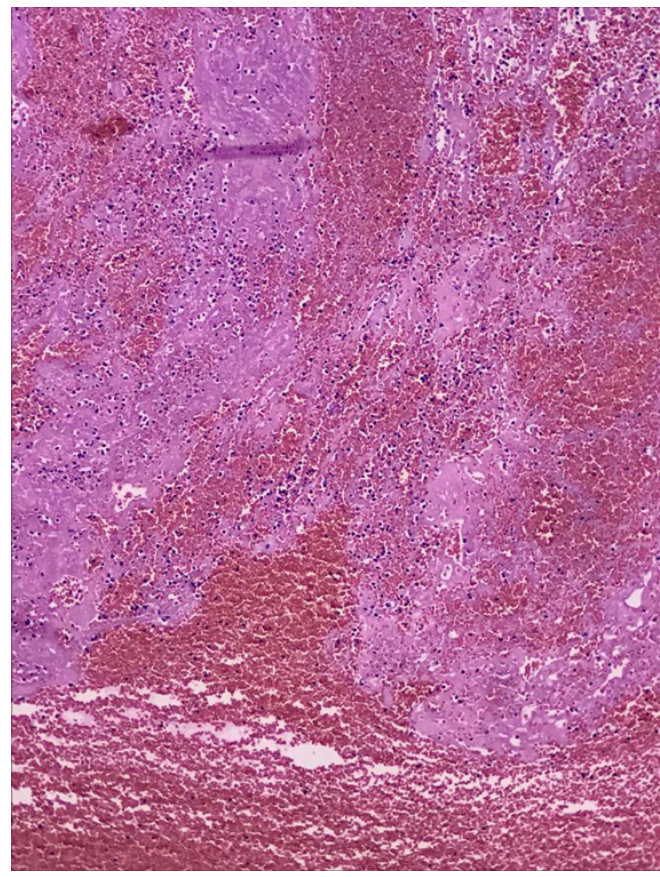

Figura 7. Análise histopatológica. Coloração H\&E: escala de x400. Trombo fibrino-hemático agudo em processo de organização.

\section{DISCUSSÃO}

Em revisão sistemática de artigos publicados em língua inglesa, foram encontrados 46 relatos de aneurisma verdadeiro isolado da $\mathrm{AFP}^{6}$. Em outra revisão no ano anterior, identificou uma série de pouco mais de 50 casos relatados ${ }^{7}$. Recentemente, aproximadamente 140 casos de aneurismas de AFP foram descritos na literatura ${ }^{11}$, mostrando que, ainda que crescente, são números que reforçam a raridade dessa entidade clínica.

Geralmente, os aneurismas de artérias femorais estão associados a aneurismas em outras topografias, como aorta e membros inferiores, e são bilaterais em 5\% dos $\operatorname{casos}^{1,4-8}$. Em $50 \%$ dos casos, há associação com DAP no território fêmoro-poplíteo ${ }^{4}$. Os aneurismas falsos e pseudoaneurismas são mais comumente encontrados na literatura, principalmente os casos pós-traumáticos e iatrogênicos ${ }^{6,12}$. Considera-se que a baixa incidência se deva a contenção oferecida pelas estruturas musculares adjacentes, em especial o músculo adutor magno, envolvendo e protegendo a artéria ${ }^{3,5-8,11}$.

Devido à dificuldade do diagnóstico clínico, principalmente quando o aneurisma apresenta-se de pequeno tamanho, são comuns os casos assintomáticos ou com sintomas inespecíficos do membro inferior, como dor, edema e sintomas neurológicos ${ }^{3-7,9,11}$. $\mathrm{O}$ achado mais frequente é a presença de massa pulsátil com ou sem dor, porém podem apresentar-se como 
achados incidentais em exames complementares ${ }^{3,6}$. Há ainda relato de diagnóstico diferencial com hérnia inguinal encarcerada, principalmente em idosos, o que pode causar consequências desastrosas caso não seja diagnosticada antes da operação ${ }^{2}$.

O risco de complicações do aneurisma de AFP é elevado, frente à dificuldade no seu diagnóstico e, portanto, ao maior tempo sem tratamento da doença, o que pode levar o paciente a apresentar inicialmente trombose, embolização distal e ruptura ${ }^{4,9}$. O risco de rotura, a complicação mais comum, varia entre 15 e $55 \%$, com associação direta do tamanho do aneurisma com o risco de rotura, sendo o diâmetro dos aneurismas da AFP geralmente maior que o da $\mathrm{AFS}^{3,6,7,13}$. A dificuldade de reconstrução devido a distorção da anatomia contribui para a elevada taxa de amputação quando os aneurismas evoluem com rotura ${ }^{4,6}$.

Na literatura, há diversas opções de abordagens cirúrgicas $^{11}$, como ligadura e ressecção isolada do aneurisma ${ }^{3,6-8,81}$ ou associadas ou à revascularização $0^{4,9} \mathrm{com}$ material sintético ou autólogo, além de procedimentos endovasculares por implante de stent recoberto ${ }^{1,13}$ e embolização, porém ainda não se dispõe de dados sobre a perviedade a longo prazo dos stents revestidos nessa topografia ${ }^{1}$. Podem ainda ser limitações para o tratamento endovascular: a extensão da doença para a AFC ou ainda a ausência de colo proximal de extensão adequada para o selamento proximal. Considera-se a ligadura segura, particularmente na presença de pulsos distais e de segmento femoropoplíteo pérvio; no entanto, todo esforço deve ser feito para a revascularização quando o pulso distal está ausente ${ }^{6,7,9,11}$. A AFP desempenha importante papel na circulação colateral do membro inferior de paciente com DAP, com progressão ao longo dos anos ${ }^{4}$.

Para estabelecer o diagnóstico de certeza, o EDC, a ATC e a angiografia são extremamente úteis. AATC de múltiplos detectores ou angiografia é especialmente recomendada para evidenciar a presença de outros aneurismas e lesões arteriais estenóticas ou oclusivas ${ }^{3,7}$. É necessário atentar-se também à anatomia e suas alterações, visto que há descrição de variações anatômicas nessa área que podem levar a desastres durante procedimentos, caso não tenham sido identificadas previamente ${ }^{14}$.

\section{CONCLUSÕES}

No caso relatado, o paciente apresentava aneurisma de AFP associada a DAP e, portanto, havia necessidade de revascularização para manutenção da circulação colateral e perfusão adequada do membro inferior. Ofereceu-se ao paciente opção terapêutica cirúrgica efetiva e segura para o tratamento da doença, sem complicações pós-operatória a curto prazo. Dessa forma, deve-se considerar que a cirurgia convencional ocupe papel de destaque no tratamento dos aneurismas acometendo a AFP.

\section{REFERÊNCIAS}

1. Okugi S, Watanabe K, Kunii Y, Koide M. Surgical and endovascular treatment of a bilateral deep femoral artery aneurysm. Interact Cardiovasc Thorac Surg. 2020;30(6):945-6. http://dx.doi.org/10.1093/ icvts/ivaa031. PMid:32170941.

2. Hamasaki T, Ota R, Matsuoka Y, Kodama W, Fukino S, Nishimura K. Ruptured deep femoral artery aneurysm confused with an incarcerated inguinal hernia: a case of an elderly patient. Int J Angiol. 2019;28(1):28-30. http://dx.doi.org/10.1055/s-0038-1676965. PMid:30880889.

3. Cho YP, Choi SJ, Kwon TW, et al. Deep femoral artery aneurysm presenting as lower limb swelling: a case report. Yonsei Med J. 2006;47(1):148-51. http://dx.doi.org/10.3349/ymj.2006.47.1.148. PMid:16502499.

4. Maruyama Y, Ochi M, Shimizu K. Surgical management of a deep femoral artery aneurysm. J Nippon Med Sch. 2012;79(5):377-80. http://dx.doi.org/10.1272/jnms.79.377. PMid:23123396.

5. Suehiro $Y$, Seo H, Kubota $Y$, Suehiro S, Hirai H. Surgical management of incidental profunda femoris artery aneurysm: a case report. Ann Vasc Surg. 2020;66:666.e11-4. http://dx.doi.org/10.1016/j. avsg.2019.12.018. PMid:31904518.

6. Posner SR, Wilensky J, Dimick J, Henke PK. A true aneurysm of the profunda femoris artery: A case report and review of the english language literature. Ann Vasc Surg. 2004;18(6):740-6. http://dx.doi. org/10.1007/s10016-004-0116-4. PMid:15599634.

7. Lima IA, Lima MF, Bernardes MV. Aneurisma isolado de artéria femoral profunda: relato de caso e revisão bibliográfica. J Vasc Bras. 2003;2(333):333-8.

8. Malek AK, Tworus R, Golebiowski $M$, et al. Deep femoral artery aneurysm. EJVES Extra. 2002;3(2):39-40. http://dx.doi.org/10.1053/ ejvx.2002.0130

9. Ikeda H, Takeo M, Murakami T, Yamamoto M. A case of deep femoral artery aneurysm. J Surg Case Rep. 2015;2015(8):rjv111. http://dx.doi.org/10.1093/jscr/rjv111. PMid:26311829.

10. Eagle KA, Berger PB, Calkins $H$, et al. ACC/AHA guideline update for perioperative cardiovascular evaluation for noncardiac surgery. Executive summary a report of the American College of Cardiology/ American Heart Association Task Force on Practice Guidelines (Committee to Update the 1996 Guidelines on Perioperative Cardiovascular Evaluation for Noncardiac Surgery). Circulation. 2002;105(10):1257-67. http://dx.doi.org/10.1161/circ.105.10.1257. PMid:11889023.

11. Jang LC, Park SS. The true deep femoral artery aneurysm: a case report. Vasc Specialist Int. 2017;33(1):40-2. http://dx.doi. org/10.5758/vsi.2017.33.1.40. PMid:28377912.

12. Cavalcante LP, Scarpari R, Queiróz TM, et al. Embolização de pseudoaneurisma com fístula arteriovenosa de artéria femoral profunda decorrente de ferimento por arma branca: relato de caso. J Vasc Bras. 2015;14(2):182-5. http://dx.doi.org/10.1590/1677-5449.0081.

13. Lewszuk A, Madycki G. Endovascular management of a giant true aneurysm of the deep femoral artery in a patient with a history of internal diseases. World J Surg Surg Res. 2019;2:1-2.

14. Kumar V, Kumar VV, Murlimanju BV, Naveen NS. Origem alta da artéria femoral profunda: relato de caso e revisão da literatura. J Vasc Bras. 2011;10(3):1-5. http://dx.doi.org/10.1590/ S1677-54492011000300010. 
Correspondência Rodrigo Gibin Jaldin

Universidade Estadual Paulista "Júlio de Mesquita Filho" - UNESP, Departamento de Cirurgia e Ortopedia, Campus de Botucatu Av. Prof. Mário Rubens Guimarães Montenegro, s/n CEP 18618-687 - Botucatu (SP), Brasil Tel.: (14) 3880-1446

E-mail: rodrigo.gibin@unesp.br

Informações sobre os autores VTRSG e NDS - Residentes de Cirurgia Vascular, Hospital das Clínicas, Faculdade de Medicina de Botucatu, Universidade Estadual "Júlio de Mesquita Filho" (UNESP).

RG) - Professor, Disciplina de Cirurgia Vascular, Faculdade de Medicina de Botucatu, Universidade Estadual "Júlio de Mesquita Filho" (UNESP).

MB - Professor Assistente Doutor, Disciplina de Cirurgia Vascular, Faculdade de Medicina de Botucatu, Universidade Estadual "Júlio de Mesquita Filho" (UNESP).

MLS - Professor Livre-Docente, Disciplina de Cirurgia Vascular, Faculdade de Medicina de Botucatu, Universidade Estadual "Júlio de Mesquita Filho" (UNESP).

RAY - Professor Colaborador, Disciplina de Cirurgia Vascular, Faculdade de Medicina de Botucatu, Universidade Estadual "Júlio de Mesquita Filho" (UNESP).

WBY - Professor Titular, Disciplina de Cirurgia Vascular, Faculdade de Medicina de Botucatu, Universidade Estadual "Júlio de Mesquita Filho" (UNESP).

Contribuições dos autores Concepção e desenho do estudo: VTRSG, RG), NDS Análise e interpretação dos dados: VTRSG, RGJ, MB, MLS, RAY, WBY Coleta de dados: VTRSG, RG), NDS Redação do artigo: VTRSG, RGJ, MB, MLS, RAY, WBY Revisão crítica do texto: VTRSG, RGJ, MB, MLS, RAY, WBY Aprovação final do artigo*: VTRSG, RG), NDS, MB, MLS, RAY, WBY Análise estatística: VTRSG, RG), NDS Responsabilidade geral pelo estudo: VTRSG, RGJ, MB, MLS, RAY, WBY

*Todos os autores leram e aprovaram a versão final submetida ao J Vasc Bras. 